Article

\title{
Infinitesimal Transformations of Locally Conformal Kähler Manifolds
}

\author{
Yevhen Cherevko ${ }^{1, *(\mathbb{D}}$, Volodymyr Berezovski ${ }^{2} \mathbb{D}$, Irena Hinterleitner ${ }^{3} \mathbb{D}$ and Dana Smetanová 4 \\ 1 Department of Economic Cybernetics and Information Technologies, Odesa National Economic University, \\ 65082 Odesa, Ukraine \\ 2 Department of Mathematics and Physics, Uman National University of Horticulture, 20300 Uman, Ukraine \\ 3 Department of Mathematics, Faculty of Civil Engineering, Brno University of Technology, \\ 60190 Brno, Czech Republic \\ 4 Department of Informatics and Natural Sciences , Faculty of Technology, Institute of Technology and \\ Business in České Budějovice, 37001 Czech Budejovice, Czech Republic \\ * Correspondence: cherevko@usa.com; Tel.: +380-971270172
}

Received: 21 June 2019; Accepted: 20 July 2019; Published: 24 July 2019

\begin{abstract}
The article is devoted to infinitesimal transformations. We have obtained that LCK-manifolds do not admit nontrivial infinitesimal projective transformations. Then we study infinitesimal conformal transformations of LCK-manifolds. We have found the expression for the Lie derivative of a Lee form. We have also obtained the system of partial differential equations for the transformations, and explored its integrability conditions. Hence we have got the necessary and sufficient conditions in order that the an LCK-manifold admits a group of conformal motions. We have also calculated the number of parameters which the group depends on. We have proved that a group of conformal motions admitted by an LCK-manifold is isomorphic to a homothetic group admitted by corresponding Kählerian metric. We also established that an isometric group of an LCK-manifold is isomorphic to some subgroup of the homothetic group of the coresponding local Kählerian metric.
\end{abstract}

Keywords: Hermitian manifold; locally conformal Kähler manifold; Lee form; diffeomorphism; conformal transformation; Lie derivative

MSC: 53C55; 53C80; 35M10

\section{Introduction}

Kählerian manifolds, because of their properties, have been used for modeling of physical processes for a long time, for instance in supersymmetric theories [1], in string theories (e.g., [2], p. 411). A manifold is called locally conformal Kähler manifold (for brevity, LCK-manifolds) if its metric is conformal to some local Kählerian metric in the neighborhood of each point of the manifold. On the other hand, one knows that conformal mappings preserve the Petrov type of a manifold [3]. The LCK-manifolds are also used for physical modeling. For instance, in [4] authors offered a'Kaluza-Klein model with spontaneous compactification, using a generalized Hopf manifold. Also, researchers use locally conformally Calabi-Yau manifolds to build $M$-theory models. According to [5] a locally conformally Calabi-Yau manifold is an LCK-manifold with a Ricci-flat metric. For example an eight-dimensional Hopf manifold admits a Ricci-flat metric, hence it may be used in a model of eleven-dimensional Supergravity.

The objects under consideration in the article are the LCK-manifolds for which $\operatorname{dim}(M)=n=2 m>2$. LCK-manifolds were explored by [6-8]. The book [9] is also worth noting as one of the most distinguished in this area. Infinitesimal conformal transformations were explored in $[10,11]$. Infinitesimal conformal transformations of complex manifolds were studied 
by Yano [12]. Transformations of LCK-manifolds were explored in [13]. The main goal of the article is also to explore transformations of LCK-manifolds.

\section{Locally Conformal Kähler Manifolds}

A Hermitian manifold $\left(M^{2 m}, J, g\right)$ is called a locally conformal Kähler manifold (LCK-manifold) if there is an open cover $\mathcal{U}=\left\{U_{\alpha}\right\}_{\alpha \in A}$ of $M^{2 m}$ and a family $\left\{\sigma_{\alpha}\right\}_{\alpha \in A}$ of $C^{\infty}$ functions $\sigma_{\alpha}: U_{\alpha} \rightarrow \mathbb{R}$ so that each local metric

$$
\hat{g}_{\alpha}=\left.e^{-2 \sigma_{\alpha}} g\right|_{U_{\alpha}}
$$

is Kählerian. An LCK-manifold is endowed with some form $\omega$, called the Lee form, which can be calculated as [14]

$$
\omega=\frac{1}{m-1} \delta \Omega \circ J \quad \text { or } \quad \omega_{i}=-\frac{2}{n-2} J_{\beta, \alpha}^{\alpha} J_{i}^{\beta},
$$

The form should be closed:

$$
d \omega=0 .
$$

One can compute covariant derivative of an almost complex structure with respect to the Levi-Civita connection of $\left(M^{2 m}, J, g\right)$ using the formula

$$
J_{i, j}^{k}=\frac{1}{2}\left(\delta_{j}^{k} J_{i}^{\alpha} \omega_{\alpha}-\omega^{k} J_{i j}-J_{j}^{k} \omega_{i}+J_{\alpha}^{k} \omega^{\alpha} g_{i j}\right) .
$$

Here and below, we denote by comma covariant differentiation with respect to the Levi-Civita connection of $\left(M^{n}, J, g\right)$.

\section{Infinitesimal Transformations of Manifolds}

Definition 1. Transformation of a manifold $M^{n}$

$$
\bar{x}^{h}=x^{h}+\epsilon \xi^{h}\left(x^{1}, x^{2}, \ldots, x^{n}\right),
$$

is called infinitesimal transformation of a manifold $M^{n}$. Vector $\xi\left(x^{1}, x^{2}, \ldots, x^{n}\right)$ is often referred to as a generator of transformation. An arbitrary small parameter $\epsilon$ is independent on $x^{i}$.

The Lie derivative of a tensor of type $(p, q) T_{j_{1} \ldots j_{q}}^{i_{1} \ldots i_{p}}$ with respect to a vector field $\xi$ may be calculated by using the formula ([15], p. 196):

$$
\mathcal{L}_{\xi} T_{j_{1} \ldots j_{q}}^{i_{1} \ldots i_{p}}=T_{j_{1} \ldots j_{q}, s}^{i_{1} \ldots i_{p}} \xi^{S}+T_{k j_{2} \ldots j_{q}}^{i_{1} \ldots i_{p}} \xi^{k}{ }_{j_{1}}+\ldots+T_{j_{1} \ldots k}^{i_{1} \ldots i_{p}} \xi^{k}{ }_{, j_{q}}^{k}-T_{j_{1} \ldots j_{q}}^{l i_{2} \ldots i_{p}} \xi^{i_{1}}{ }^{i_{1}}, \ldots-T_{j_{1} \ldots j_{q}}^{i_{1} i_{2} \ldots l} \xi^{i_{p}}, l \cdot
$$

In particular, for a metric tensor $g_{i j}$ we get

$$
\mathcal{L}_{\xi} g_{i j}=\xi_{i, j}+\xi_{j, i}
$$

If a manifold $M^{n}$ is transformed then indices of the metric tensor $\bar{g}$ of the transformed $\bar{M}^{n}$ is

$$
\bar{g}_{i j}=g_{i j}+h_{i j} \epsilon,
$$

where $h_{i j}=\mathcal{L}_{\xi} g_{i j}=\xi_{i, j}+\xi_{j, i}$, and $\epsilon$ is the arbitrary small parameter mentioned in the Definition 1 ([10], p. 275). For the Christoffel symbols we have also ([16], p. 8) :

$$
\mathcal{L}_{\xi} \Gamma_{j k}^{h}=\nabla_{k} \nabla_{j} \xi^{h}+\xi^{m} R_{j m k}^{h}
$$


Contracting (7) with $g_{h i}$ we get:

$$
\xi_{i, j k}=\xi_{\alpha} R_{k j i}^{\alpha}+g_{h i} \mathcal{L}_{\xi} \Gamma_{j k}^{h}
$$

The item $g_{h i} \mathcal{L}_{\xi} \Gamma_{j k}^{h}$ depends on transformation type. We are interested primarily in the case when a vector field $\xi\left(x^{1}, x^{2}, \ldots, x^{n}\right)$ generates a transformation preserving the complex structure [12]:

$$
\mathcal{L}_{\xi} J_{j}^{i}=J_{j, k}^{i} \xi^{k}-J_{j}^{\alpha} \xi^{i}, \alpha+J_{\alpha}^{i} \xi^{\alpha},{ }_{, j}=0 .
$$

The field is called a contravariant analytic vector field, and the infinitesimal transformation is referred to as a holomorphic one. It is worth noting that since exterior differentiation and the Lie derivation with respect to $\xi$ are commutative

$$
d \mathcal{L}_{\xi} \omega=\mathcal{L}_{\xi} d \omega
$$

hence any infinitesimal transformation preserves the closeness property of a Lee form.

\subsection{Projective Transformations and LCK-Manifolds}

If a transformation (3) does not change geodesics of a manifold, it is called a projective transformation. Mikeš and Radulovich in [7] proved that LCK-manifolds $(n>2)$ do not admit nontrivial finite geodesic mappings onto Hermitian manifolds if a preserving complex structure is required. We have to explore whether nontrivial projective transformations preserving a complex structure are admitted on LCK-manifolds. Hence let us suppose that such transformation is admitted. Then

$$
L_{\xi} \Gamma_{i j}^{h}=\psi_{i} \delta_{j}^{h}+\psi_{j} \delta_{i}^{h}
$$

where $\psi$ is a scalar whose gradient $\psi_{i}=\partial_{i} \psi$ and a vector $\xi$ generates the transformation. Then combining (8) and its conditions of integrability, we obtain:

$$
\left\{\begin{array}{l}
\xi_{i, j}=\xi_{i j} ; \\
\psi_{, i}=\psi_{i} ; \\
\xi_{i, j k}=\xi_{\alpha} R_{k j i}^{\alpha}+\psi_{k} g_{i j}+\psi_{j} g_{i k} \\
\psi_{i j}=\frac{1}{n-1}\left(\xi^{\alpha} R_{\alpha i, j}+\xi^{\alpha}{ }_{, i} R_{\alpha j}+\xi^{\alpha}{ }_{, j} R_{\alpha i}+\xi^{\alpha} R_{i j \alpha, \beta}^{\beta}\right)
\end{array}\right.
$$

Also the equation

$$
h_{i j, k}=2 \psi_{k} g_{i j}+\psi_{i} g_{j k}+\psi_{j} g_{i k}
$$

is satisfied ([10], p. 275). Since the metric $g_{i j}$ is Hermitian, we get:

$$
J_{i}^{t} g_{t j}+J_{j}^{t} g_{t i}=0 .
$$

Also, since deformed metric $\bar{g}_{i j}$ is Hermitian and the complex structure is preserved, hence on the deformed manifold $\bar{M}^{n}$, the identity

$$
J_{i}^{t} \bar{g}_{t j}+J_{j}^{t} \bar{g}_{t i}=0 .
$$

is satisfied. Taking into account (6) and (12), from (13) we obtain:

$$
J_{i}^{t} h_{t j}+J_{j}^{t} h_{t i}=0
$$

Differentiating covariantly (14) with respect to the Levi-Civita connection which is compatible with a metric $g_{i j}$, we get:

$$
J_{i, k}^{t} h_{t j}+J_{i}^{t} h_{t j, k}+J_{j, k}^{t} h_{t i}+J_{j}^{t} h_{t i, k}=0 .
$$


Then we use (2) and (11):

$$
\begin{aligned}
& \frac{1}{2}\left(\delta_{k}^{t} J_{i}^{\alpha} \omega_{\alpha}-\omega^{t} J_{i k}-J_{k}^{t} \omega_{i}+J_{\alpha}^{t} \omega^{\alpha} g_{i k}\right) h_{t j}+J_{i}^{t}\left(2 \psi_{k} g_{t j}+\psi_{t} g_{j k}+\psi_{j} g_{t k}\right) \\
& \quad+\frac{1}{2}\left(\delta_{k}^{t} J_{j}^{\alpha} \omega_{\alpha}-\omega^{t} J_{j k}-J_{k}^{t} \omega_{j}+J_{\alpha}^{t} \omega^{\alpha} g_{j k}\right) h_{t i}+J_{j}^{t}\left(2 \psi_{k} g_{t i}+\psi_{t} g_{i k}+\psi_{i} g_{t k}\right)=0 .
\end{aligned}
$$

Then, let us regroup the items:

$$
\begin{array}{r}
\left(\psi_{j}-\frac{1}{2} \omega^{t} h_{t j}\right) J_{i k}+\left(\psi_{i}-\frac{1}{2} \omega^{t} h_{t i}\right) J_{j k} \\
-\frac{1}{2} h_{s j} J_{k}^{s} \omega_{i}-\frac{1}{2} h_{s i} J_{k}^{s} \omega_{j}+\left(J_{j}^{t} \psi_{t}+\frac{1}{2} h_{t j} J_{s}^{t} \omega^{s}\right) g_{i k} \\
+\left(J_{i}^{t} \psi_{t}+\frac{1}{2} h_{t i} J_{s}^{t} \omega^{s}\right) g_{j k}+\frac{1}{2} h_{k j} J_{i}^{s} \omega_{s}+\frac{1}{2} h_{k i} J_{j}^{s} \omega_{s}=0 .
\end{array}
$$

Using symmetrization of (15), and taking into account that according to (12) and (14), the sum of the first four items in left hand side of (15) is equal to zero, we get

$$
\begin{array}{r}
\left(J_{j}^{t} \psi_{t}+\frac{1}{2} h_{t j} J_{s}^{t} \omega^{s}\right) g_{i k}+\left(J_{i}^{t} \psi_{t}+\frac{1}{2} h_{t i} J_{s}^{t} \omega^{s}\right) g_{j k} \\
+\left(J_{k}^{t} \psi_{t}+\frac{1}{2} h_{t k} J_{s}^{t} \omega^{s}\right) g_{i j}+\frac{1}{2} J_{i}^{s} \omega_{s} h_{k j}+\frac{1}{2} J_{j}^{s} \omega_{s} h_{k i}+\frac{1}{2} J_{k}^{s} \omega_{s} h_{i j}=0
\end{array}
$$

or, for brevity

$$
\chi_{j} g_{i k}+\chi_{i} g_{j k}+\chi_{k} g_{i j}+\theta_{j} h_{k i}+\theta_{i} h_{j k}+\theta_{k} h_{i j}=0,
$$

where $\chi_{i}=\left(J_{i}^{t} \psi_{t}+\frac{1}{2} h_{t i} J_{s}^{t} \omega^{s}\right), \theta_{i}=\frac{1}{2} J_{i}^{s} \omega_{s}$. If $\operatorname{dim}\left(M^{n}\right)>2$ then it is possible to choose a vector $\eta^{i}$ that $\eta^{i} \chi_{i}=\eta^{i} \theta_{i}=0$. Contracting (16) with $\eta^{i}$, we get:

$$
\chi_{j} \eta_{k}+\chi_{k} \eta_{j}+\theta_{j} h_{k i} \eta^{i}+\theta_{k} h_{i j} \eta^{i}=0 .
$$

Contracting (17) with $\eta^{j}$ produces:

$$
\chi_{k}\|\eta\|^{2}+\theta_{k} h_{i j} \eta^{i} \eta^{j}=0
$$

It follows from (18) that $\chi_{k}=\alpha \theta_{k}$. Hence,

$$
\theta_{j}\left(\alpha g_{i k}+h_{i k}\right)+\theta_{k}\left(\alpha g_{i j}+h_{i j}\right)+\theta_{i}\left(\alpha g_{j k}+h_{j k}\right)=0 .
$$

It follows from (19) that one of the equations holds, namely $\theta_{i}=0$, or $\alpha g_{j k}+h_{j k}=0$, where $\alpha$ is a certain function of the variables $x^{1}, x^{2}, \ldots, x^{n}$. In the former case we have that the manifold $M^{n}$ is Kählerian since $\omega_{i}=0$ and the transformation is trivial because $\psi_{i}=0$. In the latter case the equation $h_{j k}=-\alpha g_{j k}$ means that the transformation is a conformal one. But one knows that if a transformation is simultaneously conformal and projective then it is a trivial one. Hence we obtain the theorem.

Theorem 1. An LCK-manifold $M^{n}, \operatorname{dim}\left(M^{n}\right)>2$ does not admit nontrivial projective transformations with respect to the Levi-Civita connection preserving its complex structure.

Note that the technique we use proving the theorem is very similar to the one offered in [7].

\subsection{Conformal Infinitesimal Transformations of Locally Conformal Kähler Manifolds}

Infinitesimal transformations are called conformal if the equations hold ([12], p. 275):

$$
\mathcal{L}_{\xi} g_{i j}=\xi_{i, j}+\xi_{j, i}=\varphi g_{i j},
$$


where $\varphi$ is some function of the variables $x^{1}, x^{2}, \ldots, x^{n}$.

It is well known that, if a vector field $\xi$ generates conformal infinitesimal transformations, then the field and the invariant $\varphi$ satisfy the system $[3,11]$ :

$$
\begin{aligned}
& \text { (a) } \xi_{i, j}=\xi_{i j} ; \\
& \text { (b) } \varphi_{, i}=\varphi_{i} ; \\
& \text { (c) } \xi_{i, j}+\xi_{j, i}=\varphi g_{i j} ; \\
& \text { (d) } \xi_{i, j k}=\xi_{\alpha} R_{k j i}^{\alpha}+\frac{1}{2}\left(\varphi_{k} g_{i j}+\varphi_{j} g_{i k}-\varphi_{i} g_{j k}\right) ; \\
& \text { (e) } \varphi_{i, j}=\frac{2}{n-2}\left(\xi^{\alpha} R_{i j, \alpha}+\xi_{\alpha, i} R_{j}^{\alpha}+\xi_{\alpha, j} R_{i}^{\alpha}-\frac{g_{i j}}{2(n-1)}\left(\xi^{\alpha} R_{, \alpha}+\varphi R\right)\right) .
\end{aligned}
$$

3.3. Nijenhuis Tensor and Lee form under Conformal Infinitesimal Transformations

A necessary and sufficient condition for an almost Hermitian manifold to be Hermitian is

$$
N_{i j}^{k}=0,
$$

where

$$
N_{i j}^{k}=J_{i}^{\alpha}\left(J_{\alpha, j}^{k}-J_{j, \alpha}^{k}\right)-J_{j}^{\alpha}\left(J_{\alpha, i}^{k}-J_{i, \alpha}^{k}\right)
$$

is the Nijenhuis tensor ([12], p. 121).

The Lie derivative of the Nijenhuis tensor is

$$
\mathcal{L}_{\xi} N_{i j}^{k}=J_{i}^{\alpha}\left(\mathcal{L}_{\xi} J_{\alpha, j}^{k}-\mathcal{L}_{\xi} J_{j, \alpha}^{k}\right)-J_{j}^{\alpha}\left(\mathcal{L}_{\xi} J_{\alpha, i}^{k}-\mathcal{L}_{\xi} J_{j, \alpha}^{k}\right) .
$$

because of (9).

The following identity holds ([12], p. 159):

$$
\mathcal{L}_{\xi} J_{i, j}^{k}-\left(\mathcal{L}_{\xi} J_{i}^{k}\right)_{, j}=J_{i}^{\beta} \mathcal{L}_{\xi} \Gamma_{j \beta}^{k}-J_{\beta}^{k} \mathcal{L}_{\xi} \Gamma_{j i}^{\beta} .
$$

Because of (9), from (23) we get

$$
\mathcal{L}_{\xi} J_{i, j}^{k}=J_{i}^{\beta} \mathcal{L}_{\xi} \Gamma_{j \beta}^{k}-J_{\beta}^{k} \mathcal{L}_{\xi} \Gamma_{j i}^{\beta} .
$$

Let us calculate the Lie derivative of a Nijenhuis tensor with respect to the vector field $\xi$, taking into account (24)

$$
\begin{aligned}
\mathcal{L}_{\xi} N_{i j}^{k} & =J_{i}^{\alpha}\left(\mathcal{L}_{\mathcal{\xi}} J_{\alpha, j}^{k}-\mathcal{L}_{\xi} J_{j, \alpha}^{k}\right)-J_{j}^{\alpha}\left(\mathcal{L}_{\xi} J_{\alpha, i}^{k}-\mathcal{L}_{\xi} J_{i, \alpha}^{k}\right)= \\
= & J_{i}^{\alpha}\left(J_{\alpha}^{\beta} \mathcal{L}_{\xi} \Gamma_{j \beta}^{k}-J_{\beta}^{k} \mathcal{L}_{\xi} \Gamma_{j \alpha}^{\beta}-J_{j}^{\beta} \mathcal{L}_{\xi} \Gamma_{\alpha \beta}^{k}+J_{\beta}^{k} \mathcal{L}_{\xi} \Gamma_{\alpha j}^{\beta}\right)- \\
& -J_{j}^{\alpha}\left(J_{\alpha}^{\beta} \mathcal{L}_{\xi} \Gamma_{i \beta}^{k}-J_{\beta}^{k} \mathcal{L}_{\xi} \Gamma_{i \alpha}^{\beta}-J_{i}^{\beta} \mathcal{L}_{\xi} \Gamma_{\alpha \beta}^{k}+J_{\beta}^{k} \mathcal{L}_{\xi} \Gamma_{\alpha i}^{\beta}\right) .
\end{aligned}
$$

Removing the parentheses and collecting similar terms in (25) we obtain that the Lie derivative of a Nijenhuis tensor is equal to zero

$$
\mathcal{L}_{\tilde{\zeta}} N_{i j}^{k}=0 .
$$

Taking into account that any infinitesimal transformation preserves the closeness property of its Lee form we obtain the theorem.

Theorem 2. Any infinitesimal transformation of an LCK-manifold preserving its complex structure, transforms it into a locally conformal Kählerian one. 
Proof. Let us calculate a Lie derivative of a Lee form. Because of (9), from (1) we have

$$
\mathcal{L}_{\xi} \omega_{i}=-\frac{2}{n-2} \mathcal{L}_{\mathcal{\xi}}\left(J_{\beta, \alpha}^{\alpha} J_{i}^{\beta}\right)=-\frac{2}{n-2} \mathcal{L}_{\xi}\left(J_{\beta, \alpha}^{\alpha}\right) J_{i}^{\beta} .
$$

On the other hand, Lie derivation and contraction are commutative. Hence, contracting for $k$ and $j(24)$, we obtain

$$
\begin{array}{r}
\mathcal{L}_{\xi} J_{i, \alpha}^{\alpha}=\frac{1}{2}\left(n J_{i}^{\beta} \varphi_{\beta}-\varphi^{\alpha} J_{i \alpha}-J_{\alpha}^{\alpha} \varphi_{i}+J_{\beta}^{\alpha} \varphi^{\beta} g_{i \alpha}\right)= \\
=\frac{1}{2}\left(n J_{i}^{\beta} \varphi_{\beta}-\varphi^{\alpha} J_{i \alpha}+J_{\beta i} \varphi^{\beta}\right)= \\
=\frac{1}{2}\left(n J_{i}^{\beta} \varphi_{\beta}-\varphi^{\alpha} J_{i \alpha}-J_{i \beta} \varphi^{\beta}\right)=\frac{n-2}{2} J_{i}^{\beta} \varphi_{\beta} .
\end{array}
$$

Substituting (27) into (26) we find that

$$
\mathcal{L}_{\xi} \omega_{i}=-\frac{2}{n-2} \cdot \frac{n-2}{2} J_{\gamma}^{\beta} \varphi_{\beta} J_{i}^{\gamma}=\varphi_{i} .
$$

Theorem 3. If a vector field $\xi$ generates a conformal infinitesimal transformation of an LCK-manifold, then components of Lie derivatives of the Lee form are equal to the partial derivatives of the invariant $\varphi$ defined by the system (21)

$$
\mathcal{L}_{\xi} \omega_{i}=\varphi_{i}
$$

Proof. It is worth noting that according to (4)

$$
\mathcal{L}_{\xi} \omega_{i}=\omega_{i, \alpha} \xi^{\alpha}+\omega_{\alpha} \xi^{\alpha}{ }_{, i} .
$$

On the other hand,

$$
\frac{\partial}{\partial x^{i}}\left(\omega_{\alpha} \xi^{\alpha}\right)=\omega_{\alpha, i} \xi^{\alpha}+\omega_{\alpha} \xi^{\alpha}, i \cdot
$$

Since the Lee form is closed then $\omega_{i, j}=\omega_{j, i}$, and hence from (4) it follows that

$$
\frac{\partial}{\partial x^{i}}\left(\omega_{\alpha} \xi^{\alpha}\right)=\left(\omega_{\alpha} \xi^{\alpha}\right)_{, i}=\varphi_{i}
$$

Hence the scalar $\varphi$ mentioned in (45) may be expressed by the equation

$$
\varphi=\omega_{\alpha} \xi^{\alpha}+C,
$$

where $C$ is an arbitrary constant. Hence taking into account the conditions (9) the PDE system (21) becomes

$$
\begin{aligned}
& \text { (a) } \xi_{i, j}=\xi_{i j} ; \\
& \text { (b) } \xi_{i, j}+\xi_{j, i}=\left(\omega_{\alpha} \xi^{\alpha}+C\right) g_{i j} ; \\
& \text { (c) } \xi_{i, j k}=\xi_{\alpha} R_{k j i}^{\alpha}+\frac{1}{2}\left(\left(\omega_{\alpha} \xi^{\alpha}\right)_{, k} g_{i j}+\left(\omega_{\alpha} \xi^{\alpha}\right)_{, j} g_{i k}-\left(\omega_{\alpha} \xi^{\alpha}\right)_{, i} g_{j k}\right) ; \\
& \text { (d) } J_{j, k}^{i} \xi^{k}-J_{j}^{\alpha} \xi_{, \alpha}^{i}+J_{\alpha}^{i} \xi^{\alpha}{ }_{, j}=0 .
\end{aligned}
$$

Let us find the conditions of integrability of (30). According to ([16], p. 17) for the Levi-Civita connection the conditions are

$$
\mathcal{L}_{\xi} R_{i j k}^{h}=\nabla_{j} \mathcal{L}_{\xi} \Gamma_{i k}^{h}-\nabla_{k} \mathcal{L}_{\xi} \Gamma_{i j}^{h} .
$$

For the present case we have

$$
\mathcal{L}_{\xi} \Gamma_{i j}^{h}=\frac{1}{2}\left(\left(\delta_{i}^{h} \omega_{\alpha} \xi^{\alpha}\right)_{, j}+\delta_{j}^{h}\left(\omega_{\alpha} \xi^{\alpha}\right)_{, i} g_{i k}-g^{t h}\left(\omega_{\alpha} \xi^{\alpha}\right)_{, t} g_{i j}\right) .
$$


Since for the conformal transformations the equations

$$
\omega_{j} \mathcal{L}_{\xi} g_{i k}=\omega^{\alpha}\left(\mathcal{L}_{\xi} g_{j \alpha}\right) g_{i k},
$$

are satisfied hence (32) can be presented in the form

$$
\mathcal{L}_{\xi} \Gamma_{i j}^{h}=\mathcal{L}_{\xi} B_{i j}^{h}
$$

where

$$
B_{i j}^{h}=\frac{1}{2}\left(\delta_{i}^{h} \omega_{j}+\delta_{j}^{h} \omega_{i}-\omega^{h} g_{i j}\right) .
$$

Also there is identity ([16], p. 16) that for the present case becomes

$$
\mathcal{L}_{\xi} \nabla_{k} B_{i j}^{h}-\nabla_{k} \mathcal{L}_{\xi} B_{i j}^{h}=\mathcal{L}_{\xi} \Gamma_{t k}^{h} B_{i j}^{t}-\mathcal{L}_{\xi} \Gamma_{i k}^{t} B_{t j}^{h}-\mathcal{L}_{\xi} \Gamma_{j k}^{t} B_{i t}^{h} .
$$

Taking account of (32), (33), from (31) we obtain

$$
\mathcal{L}_{\xi} R_{i j k}^{h}=\mathcal{L}_{\xi} \nabla_{j} B_{i k}^{h}-\mathcal{L}_{\xi} \nabla_{k} B_{i j}^{h}+\mathcal{L}_{\xi}\left(B_{t k}^{h} B_{i j}^{t}\right)-\mathcal{L}_{\xi}\left(B_{t j}^{h} B_{i k}^{t}\right) .
$$

Finally we have

$$
\begin{array}{r}
\mathcal{L}_{\tilde{\xi}} R_{i j k}^{h}=\mathcal{L}_{\xi}\left(\frac{1}{2} \delta_{k}^{h} \omega_{i, j}-\frac{1}{2} \nabla_{j} \omega^{h} g_{i k}-\frac{1}{2} \delta_{j}^{h} \omega_{i, k}+\frac{1}{2} \nabla_{k} \omega^{h} g_{i j}\right. \\
+\frac{1}{4} \delta_{k}^{h} \omega_{i} \omega_{j}-\frac{1}{4} \delta_{j}^{h} \omega_{i} \omega_{k}+\frac{1}{4}\|\omega\|^{2} \delta_{j}^{h} g_{i k} \\
\left.-\frac{1}{4}\|\omega\|^{2} \delta_{k}^{h} g_{i j}+\frac{1}{4} \omega^{h} \omega_{k} g_{i j}-\frac{1}{4} \omega^{h} \omega_{j} g_{i k}\right),
\end{array}
$$

or

$$
\mathcal{L}_{\xi} Q_{i j k}^{h}=0,
$$

where $Q_{i j k}^{h}$ is defined as

$$
\begin{array}{r}
Q_{i j k}^{h}=R_{i j k}^{h}+\delta_{j}^{h}\left(\frac{1}{2} \omega_{i, k}+\frac{1}{4} \omega_{i} \omega_{k}-\frac{1}{8}\|\omega\|^{2} g_{i k}\right)-\delta_{k}^{h}\left(\frac{1}{2} \omega_{i, j}+\frac{1}{4} \omega_{i} \omega_{j}-\frac{1}{8}\|\omega\|^{2} g_{i j}\right) \\
+\left(\frac{1}{2} \omega^{h}{ }_{, j}+\frac{1}{4} \omega^{h} \omega_{j}-\frac{1}{8}\|\omega\|^{2} \delta_{j}^{h}\right) g_{i k}-\left(\frac{1}{2} \omega^{h}, k+\frac{1}{4} \omega^{h} \omega_{k}-\frac{1}{8}\|\omega\|^{2} \delta_{k}^{h}\right) g_{i j} .
\end{array}
$$

Differentiating several times (35) we get a system of differential prolongations. For convenience we use the identity for Lie derivative of tensor covariant derivative ([16], p.16) and we obtain first differential prolongation for (35)

$$
\mathcal{L}_{\xi} \nabla_{l} Q_{i j k}^{h}=\mathcal{L}_{\tilde{\zeta}} \Gamma_{t l}^{h} Q_{i j k}^{t}-\mathcal{L}_{\xi} \Gamma_{i l}^{t} Q_{t j k}^{h}-\mathcal{L}_{\xi} \Gamma_{j l}^{t} Q_{i t k}^{h}-\mathcal{L}_{\mathcal{\xi}} \Gamma_{k l}^{t} Q_{i j t}^{h},
$$

where $\mathcal{L}_{\xi} \Gamma_{j k}^{h}$ and $Q_{i j k}^{h}$ are defined by (32) and (36) respectively. We can continue the process until it turns out that the new equations are satisfied identically or the system has become inconsistent.

The Equation (30a) is solvable for $n=2 m$ unknown functions, and the Equation (30c) is solvable for $n^{2}=4 m^{2}$ unknown functions. The Equation (30b) includes $\frac{n(n+1)}{2}=\frac{2 m(2 m+1)}{2}$ restrictions. It is easy to see that (30d) determines $2 \mathrm{~m}^{2}$ independent restrictions. Since an LCK-manifold is a Hermitian one, then it follows from integrability of its almost complex structure that there exists a system of complex coordinate neighbourhoods. In the complex coordinate system $\left(z^{\alpha}, z^{\hat{\alpha}}\right)$ the condition (30d) is presented in the form

$$
\begin{aligned}
& \partial_{\hat{\beta}} \xi^{\alpha}=0 \\
& \partial_{\beta} \xi^{\hat{\alpha}}=0
\end{aligned}
$$


Hence we have

$$
\nabla_{\hat{\beta}} \xi^{\alpha}=\Gamma_{\hat{\beta} \delta}^{\alpha} \xi^{\delta}=\frac{\sqrt{-1}}{2} J_{\hat{\beta}, \delta^{\alpha}}^{\alpha} \delta^{\delta} \quad \text { and } \quad \nabla_{\beta} \xi^{\hat{\alpha}}=\Gamma_{\beta \hat{\delta}^{2}}^{\hat{\alpha} \xi^{\hat{\delta}}}=\frac{\sqrt{-1}}{2} J_{\beta, \hat{\delta}^{\hat{\delta}}}^{\hat{\delta}} .
$$

Lowering the indices we obtain

$$
\nabla_{\hat{\beta}} \xi_{\hat{\alpha}}=\frac{\sqrt{-1}}{2} J_{\hat{\beta} \hat{\alpha}, \delta} \delta^{\delta} \quad \text { and } \quad \nabla_{\beta} \xi_{\alpha}=\frac{\sqrt{-1}}{2} J_{\beta \alpha, \delta} \delta^{\hat{\delta}} .
$$

Hence we find that the Equation (30b) includes $m(m+1)$ restrictions which involve (30d). It follows that solution of the system (30) involves not more then

$$
4 m^{2}+2 m-\frac{2 m(2 m+1)}{2}+m(m+1)-2 m^{2}+1=(m+1)^{2}
$$

constants.

Theorem 4. In order for an LCK-manifold $\left(M^{n}, J, g\right)$ to admit a group of conformal transformations, it is necessary and sufficient that the equations

$$
\begin{aligned}
& \xi_{i, j}+\xi_{j, i}=\left(\omega_{\alpha} \xi^{\alpha}+C\right) g_{i j} ; \\
& J_{j, k}^{i} \xi^{k}-J_{j}^{\alpha} \xi^{i}, \alpha+J_{\alpha}^{i} \xi^{\alpha}{ }_{, j}=0,
\end{aligned}
$$

the conditions of integrability (35), their differential prolongations (37), ... etc, be algebraically consistent with respect to $\xi^{i}$ and $\xi_{j}^{i}$. If there are, among the Equations (35) and (37), ..., exactly $k$ equations which are linearly independent among themselves and of

$$
\begin{aligned}
& \xi_{i, j}+\xi_{j, i}=\left(\omega_{\alpha} \xi^{\alpha}+C\right) g_{i j} ; \\
& J_{j, k}^{i} \xi^{k}-J_{j}^{\alpha} \xi^{i}{ }_{, \alpha}+J_{\alpha}^{i} \xi^{\alpha}{ }_{, j}=0,
\end{aligned}
$$

then the LCK-manifold admits a $r=(m+1)^{2}-k$ parameter group of conformal transformations.

Considering the system (30) we can find that if $\omega_{\alpha} \xi^{\alpha}=0$, then the system may also be written in the form

$$
\begin{aligned}
& \text { (a) } \xi_{i, j}=\xi_{i j} ; \\
& \text { (b) } \xi_{i, j}+\xi_{j, i}=C g_{i j} ; \\
& \text { (c) } \xi_{i, j k}=\xi_{\alpha} R_{k j i j}^{\alpha} ; \\
& \text { (d) } J_{j, k}^{i} \xi^{k}-J_{j}^{\alpha} \xi^{i}{ }_{, \alpha}+J_{\alpha}^{i} \xi^{\alpha}{ }_{, j}=0 .
\end{aligned}
$$

Thus we have the following theorem.

Theorem 5. If on an LCK-manifold $\left(M^{n}, J, g\right)$ the Lie algebra of conformal vector fields includes such subalgebra that everywhere on $\left(M^{n}, J, g\right) \omega_{\alpha} \xi^{\alpha}=0$ holds, then the subalgebra generates a group of homothetic transformations.

Proof. The Theorem follows immediately from the Frobenius Theorem ([15], p. 201).

3.4. Local Isomorphism between Conformal Group of an LCK-Manifold and Homothetic Group of the Corresponding Kählerian Metric

Let the Kählerian metric $\hat{g}_{i j}$ be locally conformal to the metric of an LCK-manifold $\left(M^{n}, J, g\right)$. According to the definition $\hat{g}_{i j}=g_{i j} e^{-2 \sigma}, \omega_{i}=2 \sigma, i$. Then

$$
\hat{\Gamma}_{i j}^{k}=\Gamma_{i j}^{k}-\frac{1}{2} \delta_{i}^{k} \omega_{j}-\frac{1}{2} \delta_{j}^{k} \omega_{i}+\frac{1}{2} \omega^{k} g_{i j},
$$


is the Levi-Civita connection which is compatible with the metric $\hat{g}$. Let us define a contravariant vector field $\xi^{i}$ on $\left(M^{n}, J, g\right)$. Let us denote

$$
\xi_{i}=\xi^{\alpha} g_{\alpha i} ; \quad \hat{\xi}_{i}=\xi^{\alpha} \hat{g}_{\alpha i}=\xi_{i} e^{-2 \sigma} .
$$

Then we differentiate covariantly $\hat{\xi}_{i}$ with respect to the Levi-Civita connection which is compatible with the metric $\hat{g}$. Covariant derivative with respect to the connection $\hat{\Gamma}_{i j}^{k}$ is denoted as "|". Covariant derivative with respect to the connection $\Gamma_{i j}^{k}$ is denoted as usual by comma. We get

$$
\begin{aligned}
\hat{\xi}_{i \mid j} & =\hat{\xi}_{i, j}+\left(\frac{1}{2} \delta_{i}^{\alpha} \omega_{j}+\frac{1}{2} \delta_{j}^{\alpha} \omega_{i}-\frac{1}{2} \omega^{\alpha} g_{i j}\right) \hat{\xi}_{\alpha}= \\
& =\left(\xi_{i} e^{-2 \sigma}\right)_{, j}+\frac{1}{2} \hat{\xi}_{i} \omega_{j}+\frac{1}{2} \hat{\xi}_{j} \omega_{i}-\frac{1}{2} \omega^{\alpha} \hat{\xi}_{\alpha} g_{i j}= \\
& =\xi_{i, j} e^{-2 \sigma}-\xi_{i} e^{-2 \sigma} \omega_{j}+\frac{1}{2} \hat{\xi}_{i} \omega_{j}+\frac{1}{2} \hat{\xi}_{j} \omega_{i}-\frac{1}{2} \omega^{\alpha} \hat{\xi}_{\alpha} g_{i j}= \\
& =\left(\xi_{i, j}-\frac{1}{2} \xi_{i} \omega_{j}+\frac{1}{2} \xi_{j} \omega_{i}-\frac{1}{2} \omega^{\alpha} \xi_{\alpha} g_{i j}\right) e^{-2 \sigma}= \\
& =\left(\xi_{i, j}-\frac{1}{2} \xi_{i} \omega_{j}+\frac{1}{2} \xi_{j} \omega_{i}\right) e^{-2 \sigma}-\frac{1}{2} \omega^{\alpha} \xi_{\alpha} \hat{g}_{i j}
\end{aligned}
$$

Suppose that a field $\xi^{i}$ generates a homothetic group of the metric $\hat{g}$. Then it must satisfy equations

$$
\hat{\xi}_{i j j}+\hat{\xi}_{j \mid i}=C \hat{g}_{i j}
$$

Substituting (39) into (40) we obtain

$$
\begin{array}{r}
e^{-2 \sigma}\left(\xi_{i, j}+\xi_{j, i}\right)-\omega^{\alpha} \xi_{\alpha} \hat{g}_{i j}=C \hat{g}_{i j} ; \\
e^{-2 \sigma}\left(\xi_{i, j}+\xi_{j, i}\right)=C \hat{g}_{i j}+\omega^{\alpha} \xi_{\alpha} \hat{g}_{i j} ; \\
e^{-2 \sigma}\left(\xi_{i, j}+\xi_{j, i}\right)=e^{-2 \sigma}\left(\omega^{\alpha} \xi_{\alpha} g_{i j}+C g_{i j}\right) ;
\end{array}
$$

Since $e^{-2 \sigma} \neq 0$ holds, (30b) are necessarily satisfied

$$
\xi_{i, j}+\xi_{j, i}=\left(\omega_{\alpha} \xi^{\alpha}+C\right) g_{i j}
$$

Let us differentiate covariantly $\hat{\xi}_{i \mid j}$ with respect to the connection $\hat{\Gamma}_{i j}^{k}$. Since (38) holds, we obtain

$$
\begin{array}{r}
\hat{\xi}_{i \mid j k}=e^{-2 \sigma}\left(\xi_{i, j k}+\frac{1}{2}\left(\xi_{i, j}+\xi_{j, i}\right) \omega_{i}-\frac{1}{2} \omega^{\alpha}\left(\xi_{\alpha, j} g_{i k}+\xi_{i, \alpha} g_{j k}\right)\right. \\
+\frac{1}{4} \omega_{k}\left(\xi_{j} \omega_{i}-\xi_{i} \omega_{j}\right)-\frac{1}{4} \xi^{\alpha} \omega_{\alpha}\left(\omega_{i} g_{j k}-\omega_{j} g_{i k}\right) \\
+\frac{1}{2}\left(\xi_{j} \omega_{i, k}-\xi_{i} \omega_{j, k}\right)+\frac{1}{4}\|\omega\|^{2}\left(\xi_{i} g_{j k}-\xi_{j} g_{i k}\right)-\frac{1}{2}\left(\omega_{\alpha} \xi^{\alpha}\right)_{, k} g_{i j} ;
\end{array}
$$

On the other hand

$$
\begin{array}{r}
\frac{1}{2}\left(\xi_{i, j}+\xi_{j, i}\right) \omega_{i}-\frac{1}{2} \omega^{\alpha}\left(\xi_{\alpha, j} g_{i k}+\xi_{i, \alpha} g_{j k}\right) \\
=\frac{1}{2}\left(\omega_{i} \mathcal{L}_{\xi} g_{j k}-\nabla_{j}\left(\omega_{\alpha} \xi^{\alpha}\right) g_{i k}+\xi^{\alpha} \omega_{\alpha, j} g_{i k}-\omega^{\alpha}\left(\mathcal{L}_{\xi} g_{i \alpha}-\xi_{\alpha, i}\right)\right) \\
=\frac{1}{2}\left(\omega_{i} \mathcal{L}_{\xi} g_{j k}-\omega^{\alpha}\left(\mathcal{L}_{\xi} g_{i \alpha}\right) g_{j k}-\nabla_{j}\left(\omega_{\alpha} \xi^{\alpha}\right) g_{i k}\right. \\
\left.+\xi^{\alpha} \omega_{\alpha, j} g_{i k}+\nabla_{i}\left(\omega_{\alpha} \xi^{\alpha}\right) g_{j k}-\xi^{\alpha} \omega_{\alpha, i} g_{j k}\right)
\end{array}
$$


hence

$$
\begin{array}{r}
\hat{\xi}_{i \mid j k}=e^{-2 \sigma}\left(\xi_{i, j k}+\frac{1}{4} \omega_{k}\left(\xi_{j} \omega_{i}-\xi_{i} \omega_{j}\right)-\frac{1}{4} \xi^{\alpha} \omega_{\alpha}\left(\omega_{i} g_{j k}-\omega_{j} g_{i k}\right)\right. \\
+\frac{1}{2}\left(\xi_{\alpha} \omega^{\alpha}{ }_{, j} g_{i k}-\xi_{\alpha} \omega^{\alpha}{ }_{, i} g_{j k}\right)+\frac{1}{2}\left(\xi_{j} \omega_{i, k}-\xi_{i} \omega_{j, k}\right) \\
+\frac{1}{4}\|\omega\|^{2}\left(\xi_{i} g_{j k}-\xi_{j} g_{i k}\right)+\frac{1}{2}\left(\omega_{i} \mathcal{L}_{\xi} g_{j k}-\omega^{\alpha}\left(\mathcal{L}_{\xi} g_{i \alpha}\right) g_{j k}\right) \\
\left.-\frac{1}{2}\left(\left(\omega_{\alpha} \xi^{\alpha}\right)_{, k} g_{i j}+\left(\omega_{\alpha} \xi^{\alpha}\right)_{, j} g_{i k}-\left(\omega_{\alpha} \xi^{\alpha}\right)_{, i} g_{j k}\right)\right)
\end{array}
$$

Since according to (21b) in the case of conformal transformations we have $\mathcal{L}_{\xi} g_{j k}=\varphi g_{j k}$, hence $\omega_{i} \mathcal{L}_{\xi} g_{j k}-\omega^{\alpha}\left(\mathcal{L}_{\xi} g_{i \alpha}\right) g_{j k}=0$, and $(41)$ can be written as

$$
\begin{array}{r}
\hat{\zeta}_{i \mid j k}=e^{-2 \sigma}\left(\xi_{i, j k}+\frac{1}{4} \omega_{k}\left(\xi_{j} \omega_{i}-\xi_{i} \omega_{j}\right)-\frac{1}{4} \xi^{\alpha} \omega_{\alpha}\left(\omega_{i} g_{j k}-\omega_{j} g_{i k}\right)\right. \\
+\frac{1}{2}\left(\xi_{\alpha} \omega^{\alpha}{ }_{, j} g_{i k}-\xi_{\alpha} \omega^{\alpha}{ }_{, i} g_{j k}\right)+\frac{1}{2}\left(\xi_{j} \omega_{i, k}-\xi_{i} \omega_{j, k}\right) \\
\left.+\frac{1}{4}\|\omega\|^{2}\left(\xi_{i} g_{j k}-\xi_{j} g_{i k}\right)-\frac{1}{2}\left(\left(\omega_{\alpha} \xi^{\alpha}\right)_{, k} g_{i j}+\left(\omega_{\alpha} \xi^{\alpha}\right)_{, j} g_{i k}-\left(\omega_{\alpha} \xi^{\alpha}\right)_{, i} g_{j k}\right)\right),
\end{array}
$$

or

$$
\begin{array}{r}
\hat{\xi}_{i \mid j k}=e^{-2 \sigma}\left(\xi_{i, j k}+\xi_{\alpha}\left(\frac{1}{4} \omega_{k}\left(\delta_{j}^{\alpha} \omega_{i}-\delta_{i}^{\alpha} \omega_{j}\right)-\frac{1}{4} \omega^{\alpha}\left(\omega_{i} g_{j k}-\omega_{j} g_{i k}\right)\right.\right. \\
+\frac{1}{2}\left(\omega^{\alpha}{ }_{, j} g_{i k}-\omega^{\alpha}{ }_{, i} g_{j k}\right)+ \\
\left.+\frac{1}{2}\left(\delta_{j}^{\alpha} \omega_{i, k}-\delta_{i}^{\alpha} \omega_{j, k}\right)+\frac{1}{4}\|\omega\|^{2}\left(\delta_{i}^{\alpha} g_{j k}-\delta_{j}^{\alpha} g_{i k}\right)\right) \\
\left.-\frac{1}{2}\left(\left(\omega_{\alpha} \xi^{\alpha}\right)_{, k} g_{i j}+\left(\omega_{\alpha} \xi^{\alpha}\right)_{, j} g_{i k}-\left(\omega_{\alpha} \xi^{\alpha}\right)_{, i} g_{j k}\right)\right),
\end{array}
$$

where $\|\omega\|^{2}=\omega_{i} \omega_{j} g^{i j}$. On the other hand, it follows from (38) that the curvature tensor $\hat{R}$ of a Kähler metric $\hat{g}$ and the curvature tensor $R$ of an LCK-metric are related by the following expression

$$
\begin{array}{r}
\hat{R}_{i j k}^{h}=R_{i j k}^{h}+\delta_{j}^{h}\left(\frac{1}{2} \omega_{i, k}+\frac{1}{4} \omega_{i} \omega_{k}-\frac{1}{4}\|\omega\|^{2} g_{i k}\right)- \\
-\delta_{k}^{h}\left(\frac{1}{2} \omega_{i, j}+\frac{1}{4} \omega_{i} \omega_{j}-\frac{1}{4}\|\omega\|^{2} g_{i j}\right)+ \\
+\left(\frac{1}{2} \omega^{h}{ }_{j}+\frac{1}{4} \omega^{h} \omega_{j}\right) g_{i k}-\left(\frac{1}{2} \omega^{h}{ }_{, k}+\frac{1}{4} \omega^{h} \omega_{k}\right) g_{i j},
\end{array}
$$

It is known that if a field $\xi^{i}$ generates homothetic transformation of metric $\hat{g}$ then the field satisfies also the equation [10]

$$
\hat{\zeta}_{i \mid j k}=\hat{\zeta}_{\alpha} \hat{R}_{k j i}^{\alpha}
$$

Substituting (42) and (43) into (44), taking into account that $\hat{\xi}_{i}=\xi_{i} e^{-2 \sigma}$, we get

$$
e^{-2 \sigma} \xi_{i, j k}=e^{-2 \sigma}\left(\xi_{\alpha} R_{k j i}^{\alpha}+\frac{1}{2}\left(\left(\omega_{\alpha} \xi^{\alpha}\right)_{, k} g_{i j}+\left(\omega_{\alpha} \xi^{\alpha}\right)_{, j} g_{i k}-\left(\omega_{\alpha} \xi^{\alpha}\right)_{, i} g_{j k}\right)\right) .
$$

Again, it follows from $e^{-2 \sigma} \neq 0$ that (30c) is satisfied

$$
\xi_{i, j k}=\xi_{\alpha} R_{k j i}^{\alpha}+\frac{1}{2}\left(\left(\omega_{\alpha} \xi^{\alpha}\right)_{, k} g_{i j}+\left(\omega_{\alpha} \xi^{\alpha}\right)_{, j} g_{i k}-\left(\omega_{\alpha} \xi^{\alpha}\right)_{, i} g_{j k}\right)
$$

The condition that for the Kähler metric $\hat{g}$ a vector field $\xi^{i}$ satisfies

$$
\mathcal{L}_{\xi} J_{j}^{i}=J_{j \mid k}^{i} \xi^{k}-J_{j}^{\alpha} \xi_{\mid \alpha}^{i}+J_{\alpha}^{i} \xi^{\alpha}{ }_{\mid j}=0,
$$


if and only if the similar conditions (9) is satisfied. Hence if a vector field $\xi^{i}$ satisfies the system (30), then it satisfies the system

$$
\begin{aligned}
& \text { (a) } \hat{\xi}_{i, j}=\hat{\xi}_{i j ;} ; \\
& \text { (b) } \hat{\xi}_{i, j}+\hat{\xi}_{j, i}=C \hat{g}_{i j} ; \\
& \text { (c) } \hat{\xi}_{i, j k}=\hat{\xi}_{\alpha} \hat{R}_{k j i}^{\alpha} ; \\
& \text { (d) } J_{j \mid k}^{i} \xi^{k}-J_{j}^{\alpha} \xi_{\mid \alpha}^{i}+J_{\alpha}^{i} \xi^{\alpha}{ }_{\mid j}=0 .
\end{aligned}
$$

We obtain the theorem.

Theorem 6. If an LCK-manifold $\left(M^{n}, J, g\right), n=2 m$ admits a group $G_{r}$ of infinitesimal conformal transformations preserving the complex structure, then the group $G_{r}$ is isomorphic to the group of homothetic transformations of the Kähler metric $\hat{g}$ conformally corresponding to the LCK-metric.

It is worth noting that the obtained theorem is very similar to the results obtained by R. F. Bilyalov ([3], p. 274) for real Lorenzian manifolds. Namely, let $G_{r}$ be a group of conformal transformations of a Lorenzian manifold $\left(M^{n}, g\right)$ which is not conformally flat. Then we can find a manifold $\left(\hat{M}^{n}, \hat{g}\right)$, conformally corresponding to $\left(M^{n}, g\right)$ whose homothetic group is isomorphic to the group of conformal transformations of the $\left(M^{n}, g\right)$. But our result does not require that the manifold needs not to be conformally flat.

Applying the Theorems 6 and 4 to conformally flat manifolds, in particularly to a Hopf manifold, equipped by the Boothby metric, we obtain that conformal groups of the manifolds depend on $(m+1)^{2}$ parameters, where $m=\operatorname{dim}_{\mathbb{C}}\left(M^{n}\right)$.

\subsection{Conformal Infinitesimal Transformations on Compact LCK-Manifolds}

Let $\left(M^{n}, J, g\right)$ be a compact LCK-manifold, vector field $\xi$ generates conformal transformations (30b). Contracting (30c) with $g^{j k}$ we have

$$
\nabla^{t} \nabla_{t} \xi_{i}-\xi_{\alpha} R_{i}^{\alpha}=\frac{2-n}{2} \nabla_{i}\left(\omega_{\alpha} \xi^{\alpha}\right) .
$$

Then we raise the index $i$ in (45)

$$
\nabla^{t} \nabla_{t} \xi^{i}-\xi^{\alpha} R_{\alpha}^{i}=\frac{(2-n) g^{i t}}{2} \nabla_{t}\left(\omega_{\alpha} \xi^{\alpha}\right) .
$$

On the other hand, it is known [17], that a necessary and sufficient condition for a vector field $\xi$ in a compact almost Hermitian space to be contravariant almost analytic is

$$
\nabla^{t} \nabla_{t} \xi_{i}-\xi_{\alpha} R_{i}^{\alpha}=-J_{\alpha}^{i}\left(\mathcal{L}_{\xi} \nabla_{\beta} J_{\gamma}^{\beta} g^{\alpha \gamma}\right)+\frac{1}{2}\left(\nabla_{j} J_{k}^{\alpha}+\nabla_{k} J_{j}^{\alpha}\right) J_{\alpha}^{i} \mathcal{L}_{\xi} g^{j k} .
$$

For LCK-manifolds, taking account of (2) and (1), we have

$$
\begin{aligned}
-J_{\alpha}^{i}\left(\mathcal{L}_{\xi} \nabla_{\beta} J_{\gamma}^{\beta} g^{\alpha \gamma}\right)+ & \frac{1}{2}\left(\nabla_{j} J_{k}^{\alpha}+\nabla_{k} J_{j}^{\alpha}\right) J_{\alpha}^{i} \mathcal{L}_{\xi} g^{j k} \\
& =\frac{(2-n) g^{i t}}{2} \nabla_{t}\left(\omega_{\alpha} \xi^{\alpha}\right) .
\end{aligned}
$$

Comparing (46) and (47), taking account of (48) we obtain the theorem.

Theorem 7. In a compact LCK-manifold $\left(M^{n}, J, g\right)$ any vector field $\xi$ which generates nontrivial conformal transformations is contravariant almost analytic. 


\subsection{Isometries of LCK-Manifolds}

Let a vector field $\xi$ generates one-parameter continuous group of isometries of an LCK-manifold. Then the vector field $\xi$ satisfies the Killing equations.

$$
\xi_{i, j}+\xi_{i, j}=0
$$

Taking account of (39), expressing (49) with respect to the Levi-Civita connection which is compatible with the Kählerian metric $\hat{g}$, we obtain

$$
\hat{\xi}_{i \mid j}+\hat{\xi}_{j \mid i}=-\xi^{\alpha} \omega_{\alpha} \hat{g}_{i j} .
$$

But it follows from the Theorem 3 that Kählerian metric does not admit nontrivial conformal transformations. Hence $\xi^{\alpha} \omega_{\alpha}=$ const, and we obtain the theorem.

Theorem 8. Isometric Group of an LCK-manifold $\left(M^{n}, J, g\right)$ is isomorphic to some subgroup of homothetic group of the corresponding local Kählerian metric. In particular, if a vector field orthogonal to the Lee vector field is Killing with respect to the LCK-metric $g$ then the field is also Killing with respect to the local Kählerian metric $\hat{g}$.

\subsection{Transformations Generated by the Lee Fields and Anti-Lee Vector Fields on Pseudo-Vaisman Manifolds}

Let us consider a pseudo-Vaisman manifold [18], i.e., the LCK-manifold whose Lee form satisfies the equation

$$
\Phi_{4}(\nabla \omega(X, Y))=\frac{\|\omega\|^{2}}{2} g(X, Y),
$$

where $\Phi_{4}$ is the fourth Obata projector. It follows from (51) that, Lie derivative with respect to the vector field $B=\omega^{\#}$ satisfies the equations

$$
\mathcal{L}_{B} g_{i j}+J_{i}^{s}\left(\mathcal{L}_{B} g_{s t}\right) J_{j}^{t}=2\|\omega\|^{2} g_{i j} .
$$

Let us find a Lie derivative of a fundamental form $\Omega_{i j}=J_{i}^{s} g_{s j}$. According to ([9], p. 4) on an LCK-manifold, covariant derivatives of the complex structure in the directions of $B$ or $A$ are equal to zero:

$$
\nabla_{B} J=\nabla_{A} J=0 .
$$

Here $A=-J B$ is so called the anti-Lee vector field, the symbol $\nabla_{B}$ denotes the covariant derivative of the Riemannian connection defined by the LCK-metric $g$ with respect to $B$, etc. Hence

$$
\mathcal{L}_{B} \Omega_{i j}=\Omega_{t j} \nabla_{i} \omega^{t}+\Omega_{i t} \nabla_{j} \omega^{t}=-J_{j}^{t} \omega_{t, i}+J_{i}^{t} \omega_{t, j} .
$$

Since (51) is equivalent to

$$
\omega_{t, j} J_{i}^{t}-\omega_{t, i} J_{j}^{t}-\|\omega\|^{2} J_{i j}=0,
$$

it follows from (53) that

$$
\mathcal{L}_{B} \Omega_{i j}=\|\omega\|^{2} \Omega_{i j} .
$$

Let us find a Lie derivative of the fundamental form with respect to the anti-Lee vector field $A=-J B=\theta^{\#}$. Since (52) holds, we have

$$
\begin{array}{r}
\mathcal{L}_{A} \Omega_{i j}=\Omega_{t j} \nabla_{i} \theta^{t}+\Omega_{i t} \nabla_{j} \theta^{t}=J_{i}^{t} \theta_{t, j}-J_{j}^{t} \theta_{t, i} \\
=J_{i}^{t} \nabla_{j}\left(J_{t}^{s} \omega_{s}\right)-J_{j}^{t} \nabla_{i}\left(J_{t}^{s} \omega_{s}\right)=J_{i}^{t} \omega_{s} J_{t, j}^{s}-J_{j}^{t} \omega_{s} J_{t, i}^{s}+J_{i}^{t} J_{t}^{s} \omega_{s, j}-J_{j}^{t} J_{t}^{s} \omega_{s, i} \\
=-\omega_{i, j}-\omega_{j, i}+\frac{1}{2} J_{i}^{t} \omega_{s}\left(\delta_{j}^{s} J_{t}^{u} \omega_{u}-\omega^{s} J_{t j}-J_{j}^{s} \omega_{t}+J_{u}^{s} \omega^{u} g_{t j}\right) \\
-\frac{1}{2} J_{j}^{t} \omega_{s}\left(\delta_{i}^{s} J_{t}^{u} \omega_{u}-\omega^{s} J_{t i}-J_{i}^{s} \omega_{t}+J_{u}^{s} \omega^{u} g_{t i}\right) .
\end{array}
$$


Removing the parentheses in (55), and taking into account that Lee form is closed, we have

$$
\mathcal{L}_{A} \Omega_{i j}=0 .
$$

We obtain the theorem.

Theorem 9. On a pseudo-Vaisman manifold, i.e., on an LCK-manifold whose Lee form satisfies the condition

$$
\Phi_{4}(\nabla \omega(X, Y))=\frac{\|\omega\|^{2}}{2} g(X, Y),
$$

Lie derivatives of the fundamental form with respect to the Lee vector field $B=\omega^{\#}$ and to the vector anti-Lee vector field $A=-J B=\theta^{\#}$ satisfy the equations

$$
\begin{aligned}
& \text { 1) } \mathcal{L}_{B} \Omega_{i j}=\|\omega\|^{2} \Omega_{i j}, \\
& \text { 2) } \mathcal{L}_{A} \Omega_{i j}=0 .
\end{aligned}
$$

Let us find a Lie derivative of the complex structure with respect to the Lee vector field $B$ and the anti-Lee vector field $A$ taking account of (52).

$$
\begin{gathered}
\mathcal{L}_{B} J_{i}^{k}=J_{s}^{k} \nabla_{i} \omega^{s}-J_{i}^{t} \nabla_{t} \omega^{k} . \\
\mathcal{L}_{A} J_{i}^{k}=J_{t}^{k} \nabla_{i} \theta^{t}-J_{i}^{t} \nabla_{t} \theta^{k}=-J_{t}^{k} \nabla_{i}\left(J_{s}^{t} \omega^{s}\right)+J_{i}^{t} \nabla_{t}\left(J_{s}^{k} \omega^{s}\right) \\
=-J_{t}^{k} J_{s}^{t} \nabla_{i} \omega^{s}+J_{i}^{t} J_{s}^{k} \nabla_{t} \omega^{s}-J_{t}^{k} \omega^{s} J_{s, i}^{t}+J_{i}^{t} \omega^{s} J_{s, t}^{k} \\
=\nabla_{i} \omega^{k}+J_{i}^{t} J_{s}^{k} \nabla_{t} \omega^{s}-\frac{1}{2} J_{t}^{k} \omega^{s}\left(\delta_{i}^{t} J_{s}^{u} \omega_{u}-\omega^{t} J_{s i}-J_{i}^{t} \omega_{s}+J_{u}^{t} \omega^{u} g_{s i}\right) \\
+\frac{1}{2} J_{i}^{t} \omega^{s}\left(\delta_{t}^{k} J_{s}^{u} \omega_{u}-\omega^{k} J_{s t}-J_{t}^{k} \omega_{s}+J_{u}^{k} \omega^{u} g_{s t}\right) .
\end{gathered}
$$

Removing the parentheses in (57) and collecting similar terms, we obtain that

$$
\mathcal{L}_{A} J_{i}^{k}=\nabla_{i} \omega^{k}+J_{i}^{t} J_{s}^{k} \nabla_{t} \omega^{s} .
$$

Let us find a Lie derivative of the LCK-metric with respect to the anti-Lee vector field $A$

$$
\begin{array}{r}
\mathcal{L}_{A} g_{i j}=\theta_{i, j}+\theta_{j, i}=\nabla_{j}\left(J_{i}^{t} \omega_{t}\right)+\nabla_{i}\left(J_{j}^{t} \omega_{t}\right) \\
=J_{i, j}^{t} \omega_{t}+J_{i}^{t} \omega_{t, j}+J_{j, i}^{t} \omega_{t}+J_{j}^{t} \omega_{t, i} \\
=\frac{1}{2} \omega_{t}\left(\delta_{j}^{t} J_{i}^{u} \omega_{u}-\omega^{t} J_{i j}-J_{j}^{t} \omega_{i}+J_{u}^{t} \omega^{u} g_{i j}\right)+J_{i}^{t} \omega_{t, j} \\
+\frac{1}{2} \omega_{t}\left(\delta_{i}^{t} J_{j}^{u} \omega_{u}-\omega^{t} J_{j i}-J_{i}^{t} \omega_{j} i+J_{u}^{t} \omega^{u} g_{j i}\right)+J_{j}^{t} \omega_{t, i}
\end{array}
$$

Finally, we get

$$
\mathcal{L}_{A} g_{i j}=J_{i}^{t} \nabla_{j} \omega_{t}+J_{j}^{t} \nabla_{i} \omega_{t} .
$$

Now let us consider the case when the Lee form satisfies the strong pseudo-Vaisman condition

$$
\nabla \omega(X, Y)=\frac{\|\omega\|^{2}}{2} g(X, Y)
$$

Hence the Lee vector field satisfies the equations

$$
\omega_{i, j}+\omega_{j, i}=\|\omega\|^{2} g_{i j}
$$


Comparing the equations with (30b)

$$
\xi_{i, j}+\xi_{j, i}=\left(\omega_{\alpha} \xi^{\alpha}+C\right) g_{i j}
$$

we obtain, that the Lee vector field $\omega^{\#}$ generates on the LCK-manifold one-parameter conformal group for which in (30b) the condition $C=0$ holds. We get

$$
\omega_{i, j}+\omega_{j, i}=\left(\omega_{\alpha} \omega^{\alpha}\right) g_{i j}
$$

Taking account of (39) we obtain that for the connection which is compatible with the Kählerian metric $\hat{g}_{i j}=e^{-2 \sigma} g_{i j}$ the equations

$$
\hat{\omega}_{i \mid j}+\hat{\omega}_{j \mid i}=0,
$$

are satisfied. Here we note $\hat{\omega}_{i}=\omega_{t} g^{s t} \hat{g}_{t i}=e^{-2 \sigma} \omega_{i}$. It follows from (60) that the vector field $\omega^{\#}$ generates one-parameter isometry group of the Kählerian metric $\hat{g}_{i j}$. Also it follows from (56) that if the Lee form satisfies the strong pseudo-Vaisman condition, then we have

$$
\mathcal{L}_{B} J_{i}^{k}=0 .
$$

Hence the Lee vector field is contravariant analytic, i.e., a transformation generated by the field preserves the complex structure. Also, substituting the strong pseudo-Vaisman condition into (57), we obtain

$$
\mathcal{L}_{A} J_{i}^{k}=0,
$$

It means that the anti-Lee field is also contravariant analytic. Hence we write (59) in the form

$$
\mathcal{L}_{A} g_{i j}=0 .
$$

That means also that the anti-Lee vector field $\theta^{\#}$ is a Killing vector field. Taking into account Theorem 8 we make the following deductions.

Theorem 10. Let $\left(M^{n}, J, g\right)$ be an LCK-manifold, $n=2 m$ and its Lee form satisfies the strong pseudo-Vaisman condition

$$
\nabla \omega(X, Y)=\frac{\|\omega\|^{2}}{2} g(X, Y)
$$

Then:

1. The Lee and anti-Lee vector fields (respectively $\omega^{\#}$ and $\theta^{\#}$ ) are contravariant analytic.

2. On the manifold $\left(M^{n}, J, g\right)$ the Lie field $\omega^{\#}$ generates one-parameter conformal group, and anti-Lee field $\theta^{\#}$ generates one-parameter group of isometry.

3. The Lee and anti-Lee vector fields generate one-parameter isometric groups of the Kählerian metric $\hat{g}_{i j}$. The Kählerian metric $\hat{g}_{i j}$ is conformally corresponding to the LCK-metric $g$.

\section{Conclusions}

The manifolds under consideration are LCK-manifolds. The investigations use local coordinates. We assume that all functions under consideration are sufficiently differentiable, and use tensor methods (c.f. [19]).

Complex geometry deals primarily with Kählerian manifolds, i.e., manifolds carrying some Kählerian metric. Although, some complex manifolds, such as complex Hopf manifolds, admit no global Kählerian metrics at all. However, we can often find for every map of atlas a multiplyer which transforms a metric into a Kählerian one. One can say that a metric $g$ is a locally conformal Kähler (LCK) metric if $g$ is conformal to some local Kählerian metric in the neighborhood of each point of a manifold. In fact, the locally conformal Kähler manifolds were introduced by W. Westlake 
in 1954, some publications were soon made by P. Libermann, but mainly through the works of Vaisman since the 1970s has the geometry of LCK-manifolds been developed. Mappings and transformations of LCK-manifolds were explored by V. F. Kirichenko, K. Matsumoto, J. Mikeš, A. Moroianu, L. Ornea. The presented paper is devoted to infinitesimal transformations. We have obtained that LCK-manifolds does not admit nontrivial infinitesimal projective transformations. Then we study infinitesimal conformal transformations of LCK-manifolds. We have found the expression for the Lie derivative of a Lee form. We have also obtained the system of partial differential equations for the transformations, and explored its integrability conditions. Finally, we have got the necessary and sufficient conditions in order that the an LCK-manifold admit a group of conformal motions. In addition, we have calculated the number of parameters which the group depends on. We have proved that the group of conformal motions admitted by an LCK-manifold is isomorphic to the homothetic group admitted by the corresponding Kählerian metric.

Author Contributions: All authors contributed equally to this research. The research was carried out by all authors. The manuscript was prepared together and they all read and approved the final version.

Funding: This research received no external funding.

Conflicts of Interest: The authors declares no conflict of interest.

\section{References}

1. Zumino, B. Supersymmetry and Kähler Manifolds. Phys. Lett. B 1979, 87, 203-206. [CrossRef]

2. Dine, M. Supersymmetry and String Theory. Beyond the Standard Model; Cambridge University Press: Cambridge, UK, 2007; ISBN 978-0-521-85841-0.

3. Petrov, A.Z. New Methods in the General Theory of Relativity; Nauka: Moscow, Russia, 1966; p. 496.

4. Januş, S.; Visineşcu, M. Kaluza-Klein theory with scalar fields on generalized Hopf manifolds. Class. Quantum Gravty 1987, 4, 1317-1325. [CrossRef]

5. Shahbazi, C.S. M-theory on non-Kähler eight-manifolds. J. High Energy Phys. 2015, 9, 1-31. [CrossRef]

6. Kirichenko, V.F. Locally conformallity Kählerian manifolds of constant holomorphic sectional curvature. Math. USSR Sb. 1992, 72, 333-342. [CrossRef]

7. Mikeš, J.; Radulovich, Z. Geodesic mappings of locally conformal Kähler spaces. Rus. Math. 1994, 38, 48-50.

8. Vaisman, I. A geometric condition for an l.c.K. manifold to be Kähler. Geom. Dedic. 1981, 10, $129-134$. [CrossRef]

9. Dragomir, S.; Ornea, L. Locally Conformal Kähler Geometry; Birkhäuser: Boston, MA, USA; Basel, Switzerland; Berlin, Germany, 1998; ISBN 978-1-4612-2026-8.

10. Eisenhart, L.P. Riemannian Geometry; Princeton University Press: Princeton, NJ, USA, 1997; ISBN 0-691-02353-0.

11. Mikeš, J.; Moldobaev, D. Distribution of the orders of groups of conformal transformations of Riemannian spaces. Izv. Vyss. Uch. Zaved. Mat. 1991, 12, 24-29. (In Russian)

12. Yano, K. Differential Geometry on Complex and Almost Complex Spaces; Pergamon Press Book: New York, NY, USA, 1965; ISBN 008010259X.

13. Moroianu, A.; Ornea, L. Transformations of locally conformally Kähler manifolds. Manuscr. Math. 2009, 130, 93-100. [CrossRef]

14. Kirichenko, V.F. Conformally flat and locally conformal Kähler manifolds. Math. Notes 1992, 51, $462-468$. [CrossRef]

15. Dubrovin, B.A.; Fomenko, A.T.; Novikov, S.P. Modern Geometry-Methods and Applications. Part I. The Geometry of Surfaces, Transformation Groups, and Fields, 2nd ed.; Burns, R.G., Ed.; Graduate Texts in Mathematics, 93; Springer: New York, NY, USA, 1992; ISBN 0-387-97663-9.

16. Yano, K. The Theory Of Lie Derivatives And Its Applications; North-Holland Publishing Co.: Amsterdam, The Netherlands; P. Noordhoff Ltd.: Groningen, The Netherlands; Interscience Publishers Inc.: New York, NY, USA, 1957; ISBN 1296032183.

17. Yano, K.; Ako, M. Almost analytic vectors in almost complex spaces. Tohoku Math. J. 1961, 13, $24-45$. [CrossRef] 
18. Cherevko, Y.; Chepurna, O. Complex and Real Hypersurfaces of Locally Conformal Kähler Manifolds. In Proceedings of the Eighteenth International Conference on Geometry, Integrability and Quantization, Varna, Bulgaria, 3-8 June 2016; Mladenov, I.M., Meng, G., Yoshioka, A., Eds.; Avangard Prima: Sofia, Bulgaria, 2017; pp. 117-129. [CrossRef]

19. Mikeš, J.; Vanžurová, A.; Hinterleitner, I. Geodesic Mappings and Some Generalizations; Palacky University Press: Olomouc, Czech Republic, 2009; p. 304, ISBN 978-80-244-2524-5.

(C) 2019 by the authors. Licensee MDPI, Basel, Switzerland. This article is an open access article distributed under the terms and conditions of the Creative Commons Attribution (CC BY) license (http://creativecommons.org/licenses/by/4.0/). 